\title{
Time Reversal Technique based on Spatio-Temporal Windows for Through the Wall Imaging
}

DOI:

10.1109/TAP.2017.2696421

\section{Document Version}

Accepted author manuscript

Link to publication record in Manchester Research Explorer

\section{Citation for published version (APA):}

Odedo, V., Yavuz, M. E., Costen, F., Himeno, R., \& Yokota, H. (2017). Time Reversal Technique based on SpatioTemporal Windows for Through the Wall Imaging. IEEE Transactions on Antennas and Propagation, 65(6), 3065 3072. https://doi.org/10.1109/TAP.2017.2696421

\section{Published in:}

IEEE Transactions on Antennas and Propagation

\section{Citing this paper}

Please note that where the full-text provided on Manchester Research Explorer is the Author Accepted Manuscript or Proof version this may differ from the final Published version. If citing, it is advised that you check and use the publisher's definitive version.

\section{General rights}

Copyright and moral rights for the publications made accessible in the Research Explorer are retained by the authors and/or other copyright owners and it is a condition of accessing publications that users recognise and abide by the legal requirements associated with these rights.

\section{Takedown policy}

If you believe that this document breaches copyright please refer to the University of Manchester's Takedown Procedures [http://man.ac.uk/04Y6Bo] or contact uml.scholarlycommunications@manchester.ac.uk providing relevant details, so we can investigate your claim.

\section{OPEN ACCESS}




\title{
Time Reversal Technique based on Spatio-Temporal Windows for Through the Wall Imaging
}

\author{
Victor C. Odedo, Mehmet E. Yavuz, Fumie Costen, Senior Member, IEEE, Ryutaro Himeno, Hideo Yokota
}

\begin{abstract}
Time Reversal (TR) imaging methods have recently generated considerable interest due to their robustness and ability to locate multiple targets. However, problems arise when the targets are behind an obstructing material. We develop a preprocessing algorithm for TR imaging methods to obtain images of targets hidden behind a wall, applying temporal and spatial windows on the full-Multistatic Data Matrix (MDM). By using temporal-windows, we divide the full-MDM into sub-MDMs. We further segment the sub-MDMs spatially by selecting its elements corresponding to a predetermined number of antennas in space. We take the singular value decomposition of the sub-MDMs to obtain singular value distributions as well as singular vectors which we analyse individually and use for the MUltiple SIgnal Classification (MUSIC) TR imaging method. The obtained images from the these localised sub-MDMs collectively yield to clearer images of scenarios than we can obtain using the full-MDM.
\end{abstract}

Index Terms-TR, MUSIC, MDM

\section{INTRODUCTION}

$\mathbf{T}$ HE Time Reversal (TR) method which was first introduced in acoustics [1] has found applications in various disciplines ranging from non-destructive testing [2], underwater communications [3] [4] and medicine [5] [6]. TR has also been studied for Ground Penetrating Radar (GPR), Through the Wall Imaging (TWI) [7] and under lossy conditions [8] [9]. One of the main problems with GPR [10] [11] and TWI [12] [13] is performance degradation in the presence of clutter signal from an obstructing surface between the antennas and the targets. In [10], sliding spatial windows in GPR detect and localise dielectric targets shallowly buried in the ground. A study in TWI [14] is among other works [15] [16] using the target initial reflection method to locate a target hidden behind a brick wall. It was shown in [17] [18] that the MUltiple SIgnal Classification (MUSIC) algorithm can be applied to locate spatially extended targets hidden behind a wall using beamforming. A recent study used TR to locate targets behind a wall by using a single antenna, as both a transmitter and a receiver, from multiple locations, under conditions for which the clutter contribution varies minimally with antenna location [19]. Hence, the signals received are added up making the clutter signal maximum as they are in phase. However the

V. C. Odedo and F. Costen are with the School of Electrical and Electronic Engineering, The University of Manchester, U.K. (email: fumie.costen@manchester.ac.uk)

M. E. Yavuz, Hillsboro, Oregon, USA

R. Himeno is the Advanced Centre for Computing and Communication, RIKEN, Saitama, Japan

H. Yokota and F. Costen are with the Image Processing Research Team, Centre for Advanced Photonics, RIKEN, Saitama, Japan.

Color version of the figures in this paper are available online at http://ieeexplore.iee.org. configuration does not obtain a full-Multistatic Data Matrix (MDM) and hence, its application is limited. The two-stage MUSIC algorithm proposed in [20] is useful for identifying a weak targets, the responses from which have been masked due to clutter signal from an obstacle (a stronger scatterer). However the algorithm assumes having prior knowledge of the radii of the targets.

We propose a novel TR based imaging algorithm utilising simultaneously both temporal and spatial windows with which the TR-MUSIC method [21] produces better images of the medium of interest than is possible with the full-MDM in the presence of clutter. Our algorithm involves the partitioning of the elements of the full-MDM and hence the signals, to create multiple sub-MDMs which give a clearer picture of the medium than the full-MDM when used with the MUSIC method. Furthermore the spatio-temporal windows isolate the responses from targets at different times and locations in the scenario and we obtain localised information in time and space. Hence signals from the targets closer to the Time Reversal Array (TRA) antenna are contained in the earlier time-windows while signals from targets further away are contained in the later time-windows. We consider a scenario containing a target located behind a brick wall and introduce our spatio-temporal window imaging algorithm to the TR methods to locate the target and get an image of the scenario. We explain the our algorithm and discuss its application to the MUSIC methods in Section III. In Section [III] we analyse the results obtained by firstly, applying our algorithm to a scenario with a well resolved target and then a case with the target closer to the wall, where clutter and target signals overlap. Finally we emphasize the advantage of the spatial windows by applying our algorithm to a scenario with two targets.

\section{THE SPATIO-TEMPORAL WINDOW ALGORITHM}

The Decomposition Of the Time-Reversal Operator (DORT in its French acronym) and MUSIC methods [22] [23] [24] [25] are TR techniques which take advantage of the TR invariance. These methods utilise singular vectors of the MDM, whose elements $k_{s, r}(t)$, are obtained by transmitting a signal from $N$ TRA antenna elements, one at a time, and recording the response signal in the medium at all antennas, where $s$ is the transmitting antenna and $r$ is the receiving antenna. The distance between the antennas are assumed to be equally spaced. The full-MDM, $\boldsymbol{K}(t)$, which contains vital information about the targets in the medium, is represented by $\boldsymbol{K}(\omega)$ in the frequency domain. The singular value decomposition (SVD) of $\boldsymbol{K}(\omega)$ gives the singular values $\boldsymbol{\Lambda}(\omega)$ as well as 


\section{FDTD Geometry}

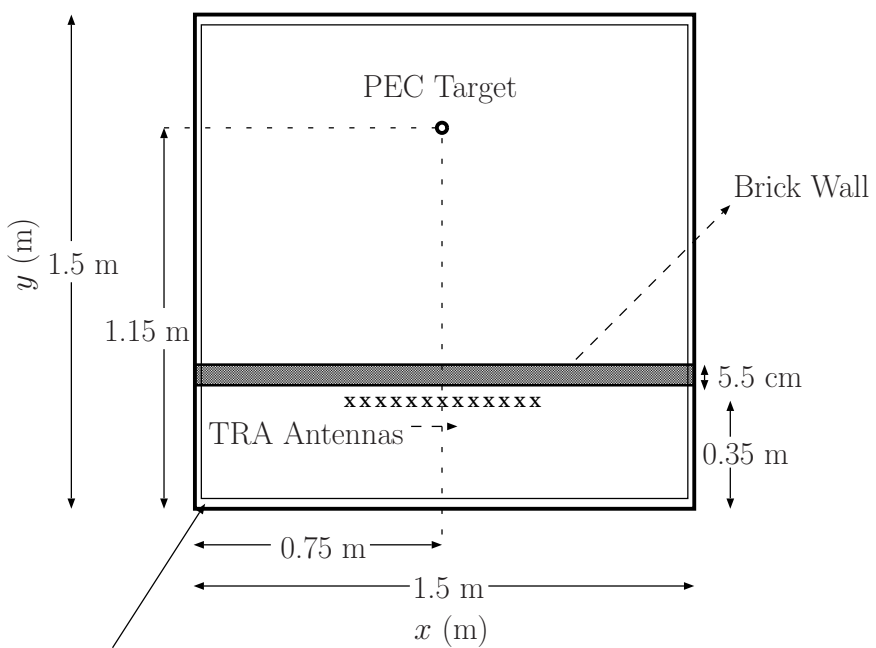

10 layer PML Region $(0.05 \mathrm{~m})$

Cell size $(\Delta x)=5 \mathrm{~mm}$

Temporal Sampling $(\Delta t)=9.6 \mathrm{ps}$

Fig. 1. The geometry of the FDTD scenario with one PEC sphere behind a brick wall

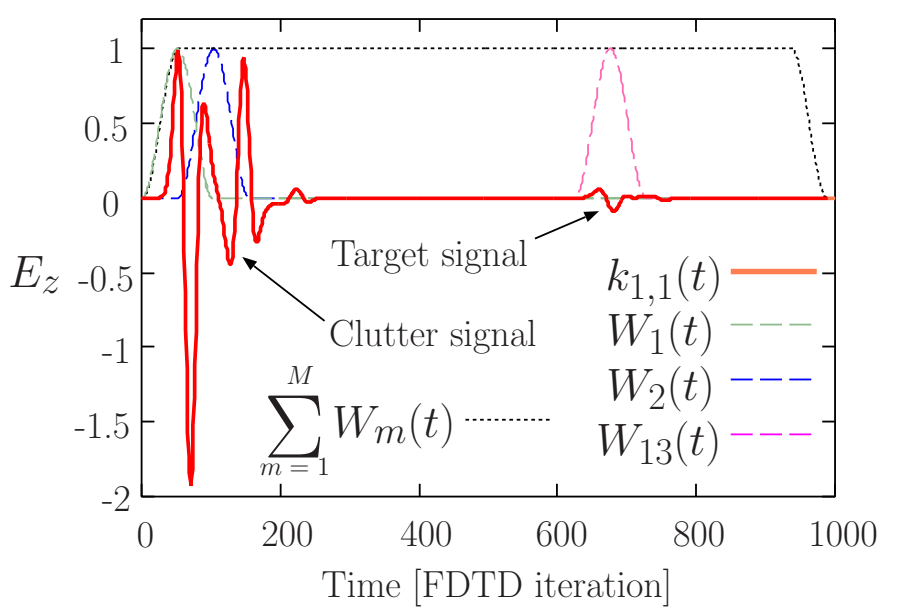

Fig. 2. The signal observed at the first antenna in Fig. 1 when a pulse was transmitted from the same antenna and line-of-sight signal is removed and the time-windows which sum to give a magnitude of 1

unitary matrices $\boldsymbol{U}(\omega)$ and $\boldsymbol{V}(\omega)$ which contain the left and right singular vectors respectively.

Due to the presence of clutter signal in $\boldsymbol{K}(t)$, the singular value distribution as well as the phase and magnitude distributions of the singular vectors obtained from SVD are greatly distorted and difficult to analyse. The reflections from the wall dominate the MDM in power which is crucial for the application of the MUSIC methods. Consequently, the ability of TR-MUSIC methods to localise the target is adversely affected. By using temporal and spatial windows, we separate the signals contained in the $\boldsymbol{K}(t)$, to multiple sub-MDMs. The SVD of the sub-MDMs gives singular values and singular vectors which we use with the TR imaging methods to create images. Hence, rather than the global information contained in the full-MDM, we obtain localised information from various parts of the scattering medium in the sub-MDMs, which reduces the number of targets to be detected at one time. Some of the benefits of localised information have already been shown in [10].

\section{A. Temporal-windowing}

We select the Hanning window [26] as our time-window. By subsequent time-shifting, we obtain temporal-windows to completely cover the whole signal duration of interest. The $m$ th time-window is obtained as

$$
W_{m}(t)=0.5\left(1-\cos \left(\frac{2 \pi\left(t-\tau_{m}\right)}{P}\right)\right)
$$

for $\frac{P(m-1)}{2} \leq t \leq P+\frac{P(m-1)}{2}$ and otherwise $W_{m}(t)=$ 0 , where $\tau_{m}$ is the time shift and $P$ is the window interval. In order to avoid loss of data, the time-windows are shifted in time from one another to ensure that the addition of the time-windows gives a magnitude of 1 . Hence, the time shift is defined as $\tau_{m}=\frac{P(m-1)}{2}(1 \leq m \leq M)$ where $M=$ $\left\lfloor\frac{T}{P \cdot 0.5}-1\right\rfloor$ is the number of time-windows needed to cover the time simulated, $T$, for the scenario, and $\lfloor\cdot\rfloor$ represents a floor function that maps a real number to the largest previous integer.

Fig. 2 shows signal $k_{1,1}(t)$ and the Hanning time-windows as well as their summation which yields a magnitude of 1 ensuring that no vital information is lost from the full-MDM. By multiplying the Hanning windows $W_{m}(t)$ by the elements of the full-MDM $k_{s, r}(t)$, one at a time, we obtain $M$ subMDMs as

$$
\begin{aligned}
\boldsymbol{K}_{m}(t)= & \left(\begin{array}{cccc}
k_{1,1}(t) W_{m}(t) & \cdot & \cdot & k_{1, N}(t) W_{m}(t) \\
\cdot & \cdot & & \cdot \\
\cdot & & \cdot & \cdot \\
k_{N, 1}(t) W_{m}(t) & \cdot & \cdot & k_{N, N}(t) W_{m}(t)
\end{array}\right) .
\end{aligned}
$$

\section{B. Space-windowing}

We further segment $\boldsymbol{K}_{m}(t)$ in space by selecting elements of $\boldsymbol{K}_{m}(t)$ obtained from a set of $N_{s}$ antennas where $1 \leq$ $N_{s} \leq N$. Hence, for $\boldsymbol{K}_{m}(t)$, we obtain the $l$ th sub-MDM as

$$
\boldsymbol{K}_{m, l}(t)=\left(\begin{array}{cccc}
k_{l, l}(t) W_{m}(t) & \cdot & \cdot & k_{l, L}(t) W_{m}(t) \\
\cdot & \cdot & & \cdot \\
\cdot & & \cdot & \cdot \\
k_{L, l}(t) W_{m}(t) & \cdot & \cdot & k_{L, L}(t) W_{m}(t)
\end{array}\right)
$$

where $L=N_{s}-1+l$ and $1 \leq l \leq N+1-N_{s}$.

\section{Setting the Window parameters}

The effectiveness of the spatio-temporal window algorithm depends on the size of the time-window $P$ and space-window $N_{s}$. A very small time-window $(P \ll$ the excitation pulse width) means that $\boldsymbol{K}_{m, l}(t)$ does not provide enough signal diversity to obtain reasonable images. However if $P$ is large enough for a single $\boldsymbol{K}_{m, l}(t)$ to contain the entire information of the full-MDM, then the algorithm reduces to the standard 
TR imaging. Assuming the excitation pulse width is $\Upsilon$, the minimum size of the time-window is chosen to be $\Upsilon$. Hence

$$
P \geq \Upsilon
$$

This ensures that a single time-window covers the initial excitation signal transmitted from the TRA antennas.

Furthermore the minimum value of $N_{s}$ for each scenario is dictated by the signal to null subspace threshold obtained from the full-MDM. Since the MUSIC methods utilise the singular vectors in the null subspace, $N_{s}$ should be greater than the number of singular values in the signal subspace $N_{t}$ of the full-MDM to ensure that we retain noise in the sub-MDMs. Therefore

$$
N_{s}>N_{t}
$$

\section{Utilising the sub-MDMs}

By performing the FFT on the elements of sub-MDM $\boldsymbol{K}_{m, l}(t)$ we obtain $\boldsymbol{K}_{m, l}(\omega)$. Furthermore the SVD on $\boldsymbol{K}_{m, l}(\omega)$ gives

$$
\boldsymbol{K}_{m, l}(\omega)=\boldsymbol{U}(\omega, m, l) \boldsymbol{\Lambda}(\omega, m, l) \boldsymbol{V}^{*}(\omega, m, l)
$$

where $\boldsymbol{\Lambda}(\omega, m, l)$ are $N_{s} \times N_{s}$ real diagonal matrices containing the singular values in descending order and, $\boldsymbol{U}(\omega, m, l)$ and $\boldsymbol{V}(\omega, m, l)$ are $N_{s} \times N_{s}$ unitary matrices containing the left and right singular vectors of $\boldsymbol{u}_{n}(\omega, m, l)$ and $\boldsymbol{v}_{n}(\omega, m, l)$ for $1 \leq n \leq N_{s}$, respectively. We know $\boldsymbol{u}_{n}(\omega, m, l)=$ $\boldsymbol{v}_{n}^{*}(\omega, m, l)$ when $\boldsymbol{K}_{m, l}(\omega)$ is symmetric [22].

The singular vectors obtained from $\boldsymbol{K}_{m, l}(\omega)$ are used to create images of the scenario using the TR-MUSIC method. The MUSIC method involve obtaining the scalar product of the singular vectors and the steering vectors $\boldsymbol{g}(\bar{r}, \omega)$. The steering vectors represent the relative phase shifts from the spatial location, $\bar{r}$, to the centre of the TRA antennas a wave experiences through the medium, where $\bar{r}=x \boldsymbol{i}+y \boldsymbol{j} . \boldsymbol{i}$ and $\boldsymbol{j}$ are unit vectors in the $x$ and $y$ directions, respectively. We assume the target is in free space. The MUSIC method utilises singular vectors in the null subspace rather than the signal subspace. The singular vectors in the signal subspace correspond to the significant singular values. The ratio of consecutive singular values corresponding to singular vectors in the same subspace (either signal or null subspace) tends to be lower than the ratio of consecutive singular values whose corresponding singular vectors are in different subspaces. Hence, for $\boldsymbol{K}(\omega)$, we can say the singular vectors, corresponding to the first $N_{t}(\omega)$ singular values, are in the signal subspace if $N_{t}(\omega)$ satisfies

$$
\frac{\lambda_{N_{t}(\omega)}(\omega)}{\lambda_{N_{t}(\omega)+1}(\omega)}=\max _{1 \leq n \leq N-1}\left(\frac{\lambda_{n}(\omega)}{\lambda_{n+1}(\omega)}\right) \text {. }
$$

We consider the Centre Frequency (CF) MUSIC and UltraWideBand (UWB) MUSIC methods [22] to utilise the singular vectors from the sub-MDMs. The MUSIC methods are capable of locating non-resolved targets as long as the number of targets is less than the number of antennas [25]. The UWB methods provide statistical stability which the $\mathrm{CF}$ methods do not in random media. By using the null subspace singular vectors from the sub-MDMs for the MUSIC methods we obtain the sub-CF-MUSIC and sub-UWB-MUSIC imaging functionals. We define the total sub-CF-MUSIC imaging functional as the summation of all sub-CF-MUSIC imaging functionals to obtain

$$
\begin{aligned}
& \boldsymbol{M}_{\Gamma}\left(\bar{r}, \omega_{c}\right)= \\
& \sum_{l=1}^{L} \sum_{m=1}^{M}\left[\sum_{n=N_{t}\left(\omega_{c}\right)+1}^{N_{s}} \boldsymbol{g}\left(\bar{r}, \omega_{c}\right) \cdot \boldsymbol{u}_{n}\left(\omega_{c}, m, l\right)\right]^{-1}
\end{aligned}
$$

where $\omega_{c}$ is the centre frequency of interest. Similarly, the summation of the sub-UWB-MUSIC imaging functionals gives the total sub-UWB-MUSIC imaging functional as

$$
\begin{aligned}
& \boldsymbol{M}_{\Gamma_{U W B}}(\bar{r})= \\
& \sum_{l=1}^{L} \sum_{m=1}^{M}\left[\int_{\Omega} \sum_{n=N_{t}(\omega)+1}^{N_{s}} \boldsymbol{g}(\bar{r}, \omega) \cdot \boldsymbol{u}_{n}(\omega, m, l) d \omega\right]^{-1}
\end{aligned}
$$

where $\Omega$ is the frequency range of interest.

\section{NUMERICAL SIMULATIONS}

\section{A. The FDTD scenario with one well-resolved target}

Fig. 1 shows the geometry of a scenario with one wellresolved PEC sphere, whose radius is $15 \mathrm{~mm}$ and conductivity is $10^{7} \mathrm{~S} / \mathrm{m}$, in a homogeneous medium (free-space) hidden behind a brick wall. A $300 \times 3002$ D FDTD space was used for the numerical simulation. The FDTD parameters for the brick wall are $0.02 \mathrm{~S} / \mathrm{m}$ for conductivity and 3.8 for relative permitivitty. The aim of this experiment is to locate the PEC sphere, assuming the location of the target is unknown despite the presence of an obstructing brick wall. A first derivative of Gaussian pulse, with a centre frequency of $2 \mathrm{GHz}$, is transmitted from $N=13$ TRA antenna elements, one at a time. The frequency range of interest is $f_{1} \leq f \leq f_{2}$ where $f_{1}$ and $f_{2}$ satisfy $S\left(f_{1}\right)=S\left(f_{2}\right)=0.1$ and $S(f)$ is the normalised spectrum of the excitation signal. The excitation used in this paper gives $f_{1}=0.3 \mathrm{GHz}$ to $f_{2}=4.8 \mathrm{GHz}$. The TRA antennas, parallel to the wall and half a wavelength at $2 \mathrm{GHz}$ apart from each other, were used to record signals which give the full-MDM $\boldsymbol{K}(t)$, containing information from the target as well as clutter signal from the brick wall. In this numerical simulation, actual antennas are not modelled but instead soft point sources [27] and observation points were used to mimic antennas. By applying the time-windows and space-windows to full-MDM $\boldsymbol{K}(t)$ we obtain sub-MDMs $\boldsymbol{K}_{m, l}(t)$. (5) with $\varsigma=\frac{\lambda}{2}$ gives $N_{s} \geq 3$.

Fig. 3 shows the singular value distribution of the fullMDM. Fig. 3. a) and (b) show the singular value distribution across the frequency range and at the centre frequency of interest, respectively. When (7) is applied to Fig. 3.b) we obtain $N_{t}\left(\omega_{c}\right)=2$ which is used in (8).

Fig. 4 shows the CF-MUSIC and UWB-MUSIC (from 0.3 to $4.8 \mathrm{GHz}$ ) images obtained for the scenario in Fig. 1 using the full-MDM while Fig. 5 shows the total sub-CF-MUSIC and total sub-UWB-MUSIC (from 0.3 to $4.8 \mathrm{GHz}$ ) images when $P=\Upsilon$ and $N_{s}=7$. 


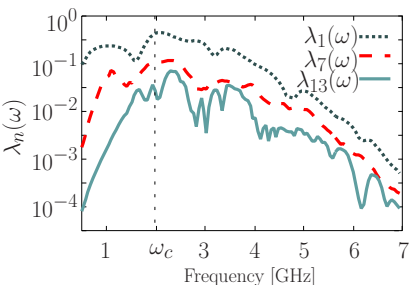

(a)

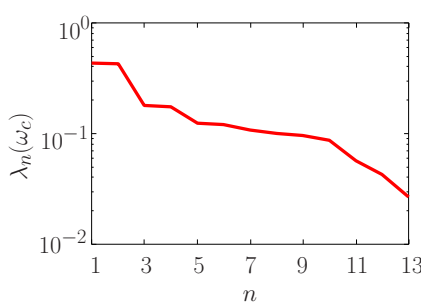

(b)
Fig. 3. Singular value distribution of the full-MDM for the scenario in Fig. 1 (a) over the frequency range of interest, (b) at the centre frequency $\omega_{c}$

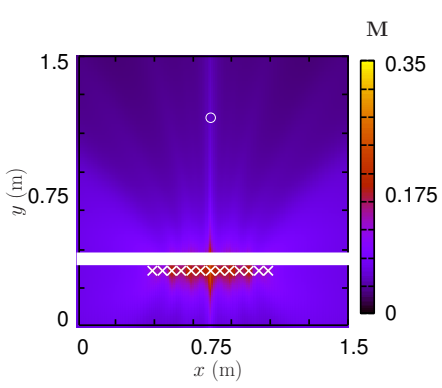

(a) CF-MUSIC



(b) UWB-MUSIC
Fig. 4. The CF-MUSIC and UWB-MUSIC images produced using the fullMDM, where $\times$ represents the TRA antennas' locations and $\circ$ represents the target location



(a) CF-MUSIC



(b) UWB-MUSIC
Fig. 5. The total sub-CF-MUSIC and total sub-UWB-MUSIC images produced when $P=\Upsilon$ and $N_{s}=7$, where $\times$ represents the TRA antennas' locations and $\circ$ represents the target location

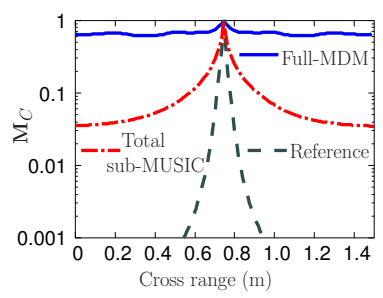

(a) CF-MUSIC



(b) UWB-MUSIC
Fig. 6. The normalised cross section at $y=1.15 \mathrm{~m}$ in Fig. 4 and Fig. 5 obtained using the full-MDM MUSIC method, the total sub-MUSIC method and the reference

Fig. 6 shows the cross section of Fig. 4 and Fig. 5 obtained using the full-MDM MUSIC method, the total sub-MUSIC

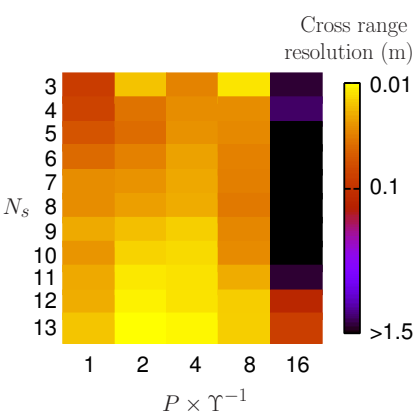

(a) CF-MUSIC

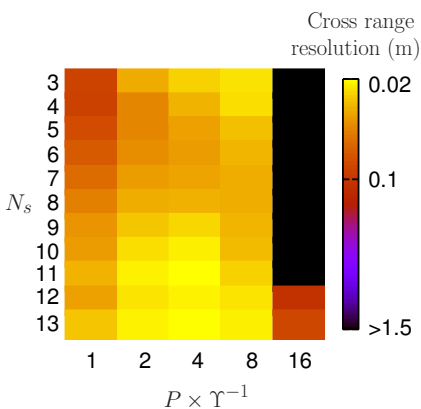

(b) UWB-MUSIC
Fig. 7. The total sub-CF-MUSIC and total sub-UWB-MUSIC cross range resolution at half maximum of $M_{\Gamma}\left(\bar{r}, \omega_{c}\right)$ and $M_{\Gamma_{U W B}}(\bar{r})$ at $y=1.15 \mathrm{~m}$, varying $N_{s}$ and $P$

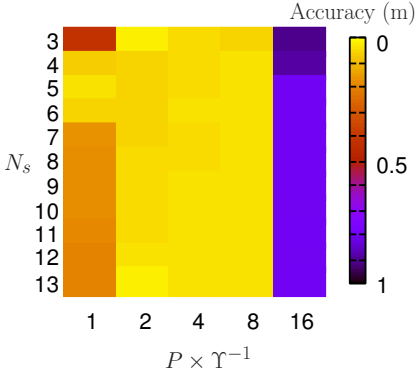

(a) CF-MUSIC

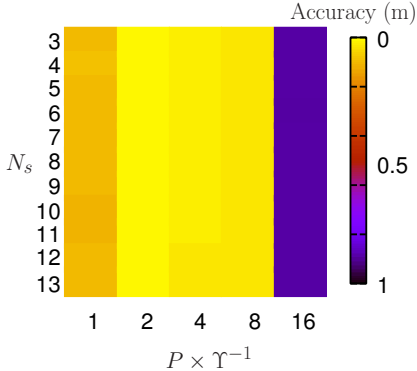

(b) UWB-MUSIC
Fig. 8. The accuracy of the total sub-CF-MUSIC and total sub-UWB-MUSIC imaging functional in locating the PEC target when $N_{s}$ and $P$ are varied

method and the cross section of the image of a reference. We obtain the reference by implementing standard TR imaging with the full-MDM on a scenario similar to Fig. 1 but without a wall. Hence, the reference has the best possible resolution attainable for the MUSIC methods. As in Fig. 4 the full-MDM did not detect the target. However the total sub-CF-MUSIC and total sub-UWB-MUSIC imaging functional yield images that accurately locate the target despite the loss of resolution from the reduced TRA antenna aperture and time-windows.

Fig. 7 shows the cross range resolution of the total sub-CFMUSIC and total sub-UWB-MUSIC images at $y=1.15 \mathrm{~m}$, varying $N_{s}$ and $P$. We obtain the cross range resolution at half maximum of $\boldsymbol{M}_{\Gamma}\left(\bar{r}, \omega_{c}\right)$ and $\boldsymbol{M}_{\Gamma_{U W B}}(\bar{r})$ at the corresponding cross section. Fig. 8 shows the accuracy of the total sub-CFMUSIC and total sub-UWB-MUSIC images in locating the PEC target, varying $N_{s}$ and $P$. We define the accuracy as the distance between the location of the maximum of $\boldsymbol{M}_{\Gamma}\left(\bar{r}, \omega_{c}\right)$ or $M_{\Gamma_{U W B}}(\bar{r})$ and the actual location of the PEC target. We observe that we retain the accuracy and the resolution of the images as we initially increase the size of the time-windows $P$. However, when $P$ gets large enough for a single sub-MDM to contain the entire information of the full-MDM, the algorithm reduces to the standard TR imaging and we are unable to locate the target due to clutter. We observe from Fig. 7 that for a scenario with a single PEC target we attain higher resolution as we increase size of the spatial windows $N_{s}$ due to the increase in aperture. However the advantage of spatial windows is 

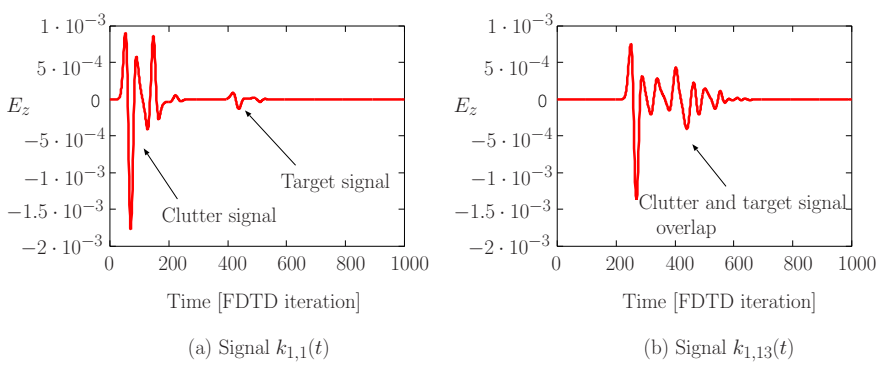

Fig. 9. Signals observed at (a) the first antenna when a pulse was transmitted from the same antenna $\left(k_{1,1}(t)\right)$ and (b) the thirteenth antenna when a pulse was transmitted from the first antenna $\left(k_{1,13}(t)\right)$ with the line-of-sight signal removed



(a) CF-MUSIC

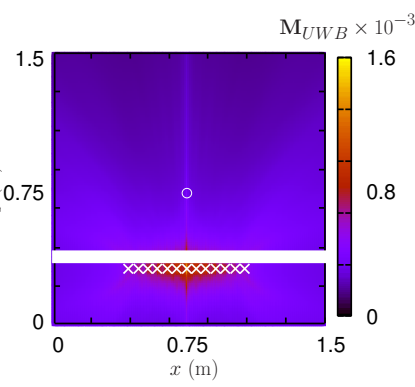

(b) UWB-MUSIC
Fig. 10. The CF-MUSIC and UWB-MUSIC images produced using the fullMDM, where $\times$ represents the TRA antennas' locations and $\circ$ represents the target location

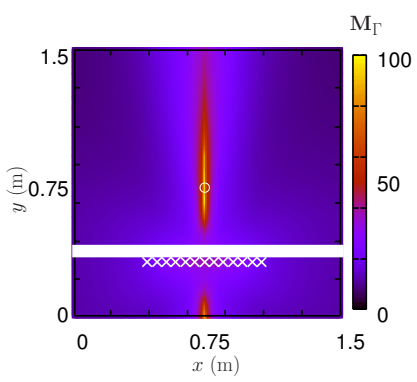

(a) CF-MUSIC

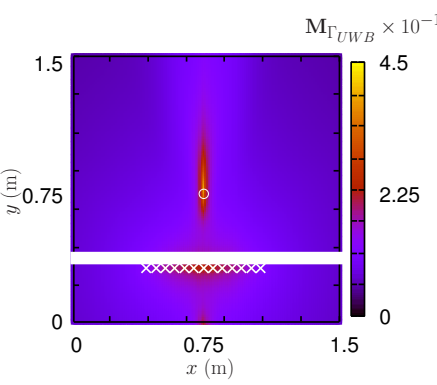

(b) UWB-MUSIC
Fig. 11. The total sub-CF-MUSIC and total sub-UWB-MUSIC images produced when $P=\Upsilon$ and $N_{s}=7$, where $\times$ represents the TRA antennas' locations and $\circ$ represents the target location

emphasized in scenarios containing multiple targets as shown in Section $\amalg I-C$.

\section{B. Scenario with clutter and target signal overlapped}

We simulate a scenario, with overlap between clutter signal and target signal in the full-MDM, by moving the target, shown in Fig. 1. $0.4 \mathrm{~m}$ towards the brick wall. Fig. 9 shows $k_{1,1}(t)$ and $k_{1,13}(t)$ from the full-MDM obtained from the scenario with clutter and target signal overlapped. The spatiotemporal windows are applied to the full-MDM to obtain subMDMs $\boldsymbol{K}_{m, l}(t)$.

Fig. 10 shows the CF-MUSIC and UWB-MUSIC (from 0.3 to $4.8 \mathrm{GHz}$ ) images obtained for the scenario using the full-
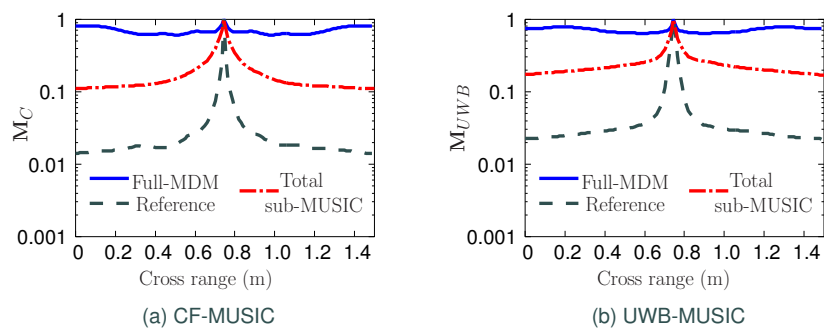

Fig. 12. The normalised cross section at $y=0.75 \mathrm{~m}$ in Fig. 10 and Fig. 11 obtained using the full-MDM MUSIC method, the total sub-MUSIC method and the reference

\section{FDTD Geometry}

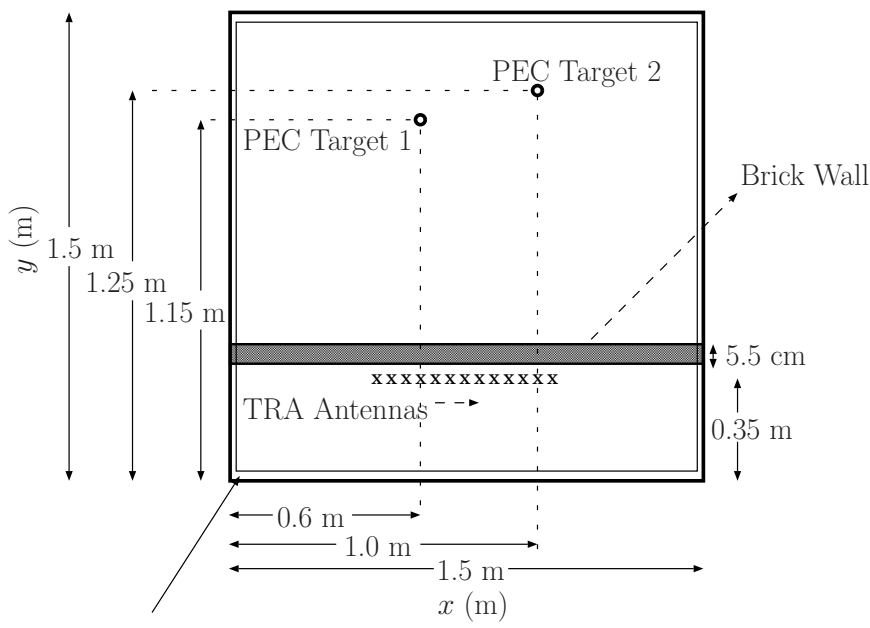

10 layer PML Region $(0.05 \mathrm{~m})$

Cell size $(\Delta x)=5 \mathrm{~mm}$ Temporal Sampling $(\Delta t)=9.6 \mathrm{ps}$

Fig. 13. The geometry of the FDTD scenario with two PEC sphere behind a brick wall

MDM. Fig. 11 shows the total sub-CF-MUSIC and total subUWB-MUSIC (from 0.3 to $4.8 \mathrm{GHz}$ ) images obtained for the scenario, with clutter and target signal overlap, when $P=\Upsilon$ and $N_{s}=7$.

Fig. 12 shows the cross section of Fig. 10 and Fig. 11 obtained using the full-MDM MUSIC method, the total subMUSIC method and the cross section of the image of a reference. The total sub-MUSIC functionals give images that accurately locate the target.

\section{Scenario with two targets}

The advantage of spatial windows is emphasized when we apply the temporal and spatial window imaging algorithm to a scenario containing multiple targets. Fig. 13 shows the geometry of a scenario with two well-resolved PEC spheres in a homogeneous medium (free-space) hidden behind a brick wall.

Fig. 14 shows the total sub-CF-MUSIC and total subUWB-MUSIC (from 0.3 to $4.8 \mathrm{GHz}$ ) images obtained for the scenario in Fig. 13 when $P=8 \cdot \Upsilon$ and $N_{s}=7$. The images locate the targets which is not achieved by the Full-MDM.

Fig. 15 and Fig. 16 shows the cross range resolution of the total sub-CF-MUSIC and total sub-UWB-MUSIC images at 


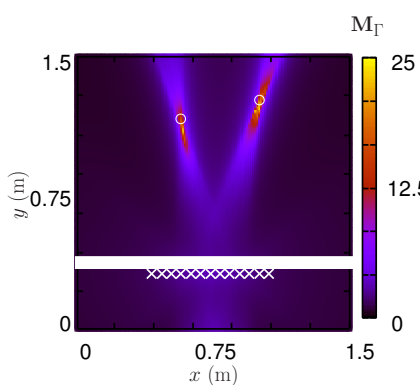

(a) CF-MUSIC

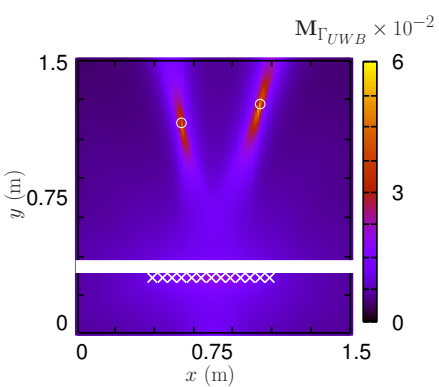

(b) UWB-MUSIC
Fig. 14. The total sub-CF-MUSIC and total sub-UWB-MUSIC images produced when $P=8 \cdot \Upsilon$ and $N_{s}=7$, where $\times$ represents the TRA antennas' locations and $\circ$ represents the location of targets

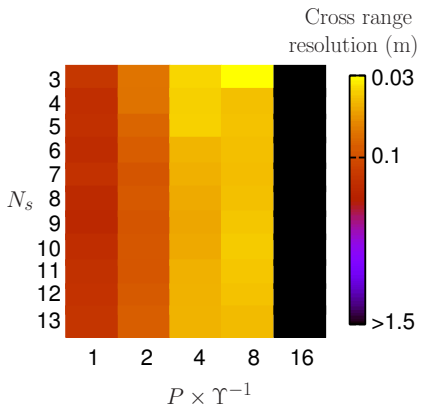

(a) CF-MUSIC

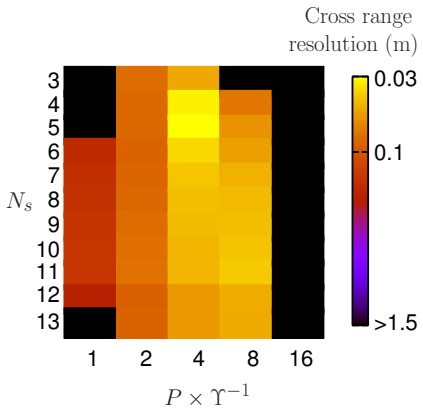

(b) UWB-MUSIC
Fig. 15. The total sub-CF-MUSIC and total sub-UWB-MUSIC cross range resolution at half maximum of $\boldsymbol{M}_{\Gamma}\left(\bar{r}, \omega_{c}\right)$ and $\boldsymbol{M}_{\Gamma_{U W B}}(\bar{r})$ at $y=1.15 \mathrm{~m}$, varying $N_{s}$ and $P$ (a) CF-MUSIC

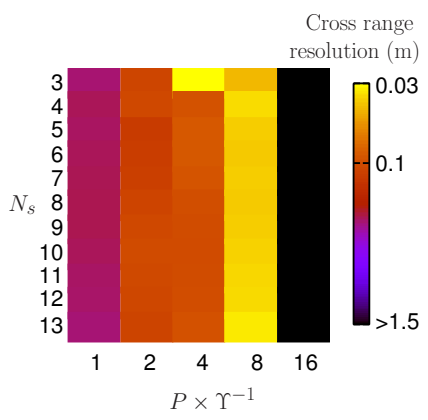

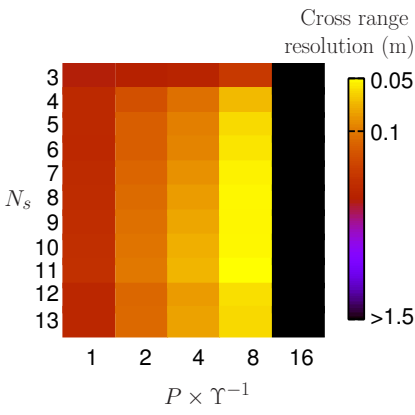

(b) UWB-MUSIC
Fig. 16. The total sub-CF-MUSIC and total sub-UWB-MUSIC cross range resolution at half maximum of $M_{\Gamma}\left(\bar{r}, \omega_{c}\right)$ and $M_{\Gamma_{U W B}}(\bar{r})$ at $y=1.25 \mathrm{~m}$, varying $N_{s}$ and $P$

$y=1.15 \mathrm{~m}$ where PEC target 1 is located and at $y=1.25 \mathrm{~m}$ where PEC target 2 is located, varying $N_{s}$ and $P$. We observe that as we increase $P$, we retain the resolution of the images until the algorithm reduces to standard TR problems. Fig. 15.a) shows that we obtain the highest resolution for PEC target 1 when $N_{s}=3$ and $P=8 \cdot \Upsilon$ for the total sub-CFMUSIC image while Fig. 15.b) shows that we obtain the highest resolution when $N_{s}=5$ and $P=4 \cdot \Upsilon$ for the total sub-UWB-MUSIC image. Similarly, Fig. 16.a) shows that we obtain the highest resolution for PEC target 2 when $N_{s}=3$ and $P=4 \cdot \Upsilon$ for the total sub-CF-MUSIC image

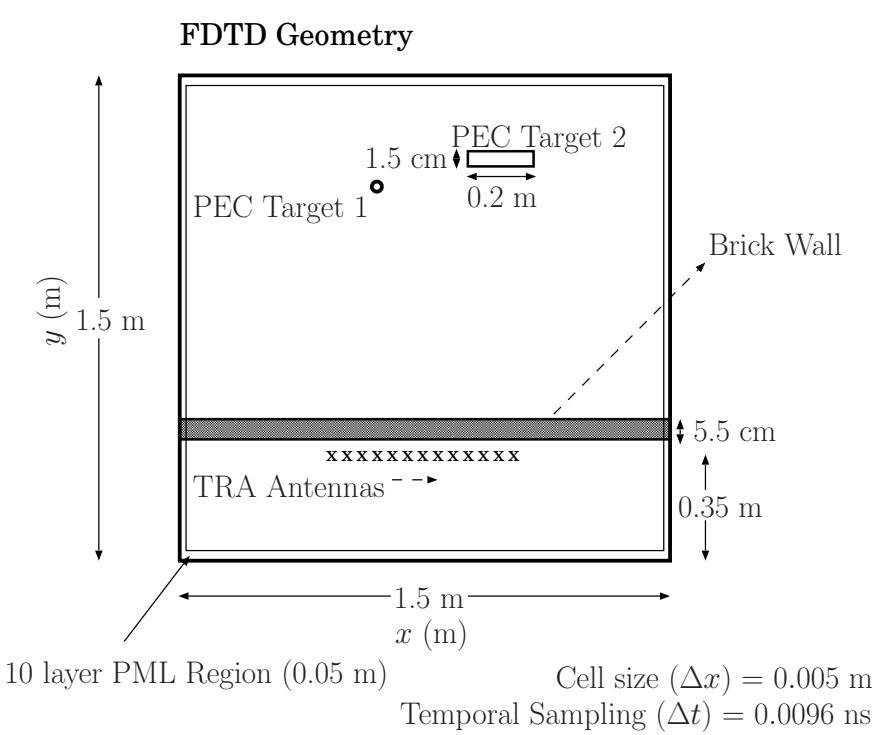

Fig. 17. The geometry of the FDTD scenario with one PEC sphere and one extended target behind a brick wall



(a)

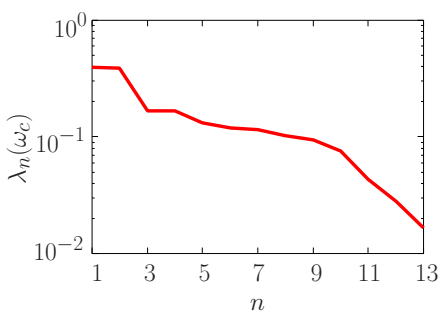

(b)
Fig. 18. Singular value distribution of the full-MDM for the scenario in Fig. 17] (a) over the frequency range of interest, (b) at the centre frequency $\omega_{c}$

while Fig. 16(b) shows that we obtain the highest resolution when $N_{s}=11$ and $P=8 \cdot \Upsilon$ for the total sub-UWBMUSIC image. Hence, Fig. 15 and Fig. 16 demonstrate that we can locate the targets with increased resolution, when the time windowed sub-MDMs are further segmented by spatial windows, despite the reduction of the TRA antenna aperture as a negative effect. The spatial windows further segregate the signals from different target into different sub-MDMs, leading to images which more accurately represent the scenario.

Fig. 17 shows the geometry of a scenario identical to Fig. 13 containing one PEC sphere whose radius is $15 \mathrm{~mm}$ and one extended PEC target added, whose length is $0.2 \mathrm{~m}$ and width is $1.5 \mathrm{~cm}$, in a homogeneous medium (free-space) hidden behind a brick wall. Fig. 18 shows the singular value distribution of the full-MDM obtained for the scenario in Fig. 17 Fig. 18,a) and (b) show the singular value distribution across the frequency range and at the centre frequency of interest, respectively. Although extended scatterers in freespace tend to produce a smooth change in the singular values, the presence of the wall, which is also an extended scatterer, close to the TRA antennas dominates the singular value distribution 


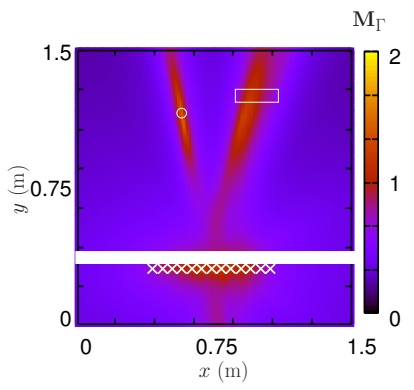

(a) CF-MUSIC

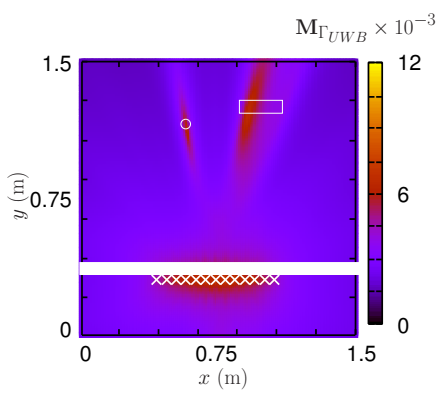

(b) UWB-MUSIC
Fig. 19. The total sub-CF-MUSIC and total sub-UWB-MUSIC images produced when $P=8 \cdot \Upsilon$ and $N_{s}=11$, where $\times$ represents the TRA antennas' locations and $\circ$ and $\square$ represent the location of targets

regardless of the targets behind the wall. Hence by applying (7) to Fig. 18(b) we obtain $N_{t}\left(\omega_{c}\right)=2$ which is used in (8). Fig. 19 shows the total sub-CF-MUSIC and total subUWB-MUSIC (from 0.3 to $4.8 \mathrm{GHz}$ ) images obtained for the scenario in Fig. 17 when $P=8 \cdot \Upsilon$ and $N_{s}=11$. We observe from the images that our algorithm locates the PEC sphere and the presence of an extended target which is not achieved using the Full-MDM for the MUSIC method.

\section{CONCLUSION}

The TR-MUSIC imaging methods suffer in performance when there is significant clutter in the full-MDM due to an obstruction between the TRA antennas and the target. We developed and applied a spatio-temporal window algorithm to extract information on targets by segmenting the full-MDM into sub-MDMs. Using sub-MDMs, we obtained images that localised the targets hidden behind a brick wall which is not possible through the classical utilisation of the full-MDM.

In the future, more complex scenarios containing multiple targets with a wall containing metallic rebars can be considered as they produce extra scattering which may benefit the resolution by enhancing the effective aperture [28]. Additionally, the impact of randomness in the background medium and dispersion in the wall media can be considered as an extension of the current work.

\section{REFERENCES}

[1] M. Fink, C. Prada, F. Wu, and D. Cassereau, "Self focusing in inhomogeneous media with time-reversal acoustics mirrors," in Ultrasonics Symposium, 1989. Proceedings., IEEE 1989, vol. 2, pp. 681-686, Oct. 1989.

[2] N. Chakroun, M. Fink, and F. Wu, "Time reversal processing in ultrasonic nondestructive testing," IEEE Trans. Ultrason., Ferroelect., Freq. Contr., vol. 42, pp. 1087-1098, 1995.

[3] S. Kim, W. A. Kuperman, W. S. Hodgkiss, H. C. Song, G. Edelman, and T. Akal, "Echo-to-reverberation enhancement using a time reversal mirror," The Journal of the Acoustical Society of America, vol. 115, pp. 1525-1531, 2004.

[4] H. C. Song, W. S. Hodgkiss, W. A. Kuperman, P. Roux, T. Akal, and M. Stevenson, "Experimental demonstration of adaptive reverberation nulling using time reversal," The Journal of the Acoustical Society of America, vol. 118, pp. 1381-1387, 2005.

[5] J. L. Thomas, F. Wu, and M. Fink, "Time reversal focusing applied to lithotripsy," Ultrason. Imag., vol. 18, pp. 106-121, 1996.

[6] P. Kosmas and C. M. Rappaport, "Time reversal with the FDTD method for microwave breast cancer detection," IEEE Trans. Microwave Theory Tech., vol. 53, pp. 2317-2323, 2005.
[7] M. E. Yavuz, "Through-the-wall sensing using time-reversal antenna arrays," in Antennas and Propagation (EuCAP), 2010 Proceedings of the Fourth European Conference on, pp. 1-4, April 2010.

[8] A. M. Abduljabbar, M. E. Yavuz, F. Costen, R. Himeno, and H. Yokota, "Frequency dispersion compensation through variable window utilization in time-reversal techniques for electromagnetic waves," IEEE Transactions on Antennas and Propagation, vol. 64, pp. 3636-3639, Aug 2016.

[9] A. M. Abduljabbar, M. E. Yavuz, F. Costen, R. Himeno, and H. Yokota, "Continuous wavelet transform based frequency dispersion compensation method for electromagnetic time-reversal imaging," IEEE Transactions on Antennas and Propagation, vol. PP, no. 99, pp. 1-1, 2017.

[10] M. E. Yavuz, A. E. Fouda, and F. L. Teixeira, "GPR signal enhancement using sliding-window space-frequency matrices," Progress In Electromagnetics Research, vol. 145, pp. 1-10, 2014.

[11] T. Dogaru and L. Carin, "Time-domain sensing of targets buried under a rough air-ground interface," IEEE Trans. Antennas and Propagat., vol. 46, pp. 360-372, 2009.

[12] W. Zhang, A. Hoorfar, and L. Li, "Through-the-wall target localization with time reversal MUSIC method," Progress In Electromagnetics Research, vol. 106, pp. 75-89, 2010.

[13] P. Rasekh, M. Razavian, A. Torki, and R. Safian, "Through the wall imaging based on time reversal independent component analysis," in 2014 IEEE Antennas and Propagation Society International Symposium (APSURSI), pp. 1117-1118, July 2014.

[14] A. B. Gorji and B. Zakeri, "An improved time-reversal-based target localization for through-wall microwave imaging," Journal of Electrical and Computer Engineering Innovations, vol. 1, pp. 89-97, 2013.

[15] P. C. Chang, Physics-based inverse processing and multipath exploitation for through-wall radar imaging. $\mathrm{PhD}$ thesis, The Ohio State University, 2011.

[16] M. Davy, T. Lepetit, J. d. Rosny, C. Prada, and M. Fink, "Detection and imaging of human beings behind a wall using the DORT method," Progress In ELectromagnetics Research, vol. 110, pp. 353-369, 2010.

[17] Y. S. Yoon and M. G. Amin, "High-resolution through-the wall radar imaging using beamspace MUSIC," IEEE Trans. Antennas and Propagat., vol. 56, pp. 1763-1774, 2008.

[18] W. Zhang, A. Hoorfar, C. Thajudeen, and F. Ahmad, "Full polarimetric beam-forming algorithm for through-the-wall radar imaging," Radio Science, vol. 46(5), 2011.

[19] R. Solimene and A. Cuccaro, "Front wall clutter rejection methods in TWI," IEEE Geoscience and remote sensing letters, vol. 11, pp. 11581162, 2014.

[20] R. Solimene, G. Leone, and A. Dell'Aversano, "MUSIC algorithms for the rebar detection," Journal of Geophysics and Engineering, vol. 10, p. 064006, 2013.

[21] H. Lev-Ari and A. J. Devancy, "The time-reversal technique reinterpreted: subspace-based signal processing for multi-static target location," in Sensor Array and Multichannel Signal Processing Workshop. 2000. Proceedings of the 2000 IEEE, pp. 509-513, 2000.

[22] M. E. Yavuz, Time Reversal Based Signal Processing Techniques For Ultrawideband Electromagnetic Sensing In Random Media. PhD thesis, The Ohio State University, 2008.

[23] M. E. Yavuz and F. L. Teixeira, "On the sensitivity of time-reversal imaging techniques to model perturbations," IEEE Trans. Antennas and Propagat., vol. 56, no. 3, pp. 834-843, 2008.

[24] M. E. Yavuz and F. L. Teixeira, "Full time-domain dort for ultrawideband electromagnetic fields in dispersive, random inhomogeneous media," IEEE Transactions on Antennas and Propagation, vol. 54, pp. 23052315, Aug 2006.

[25] R. Solimene and A. Dell'Aversano, "Some remarks on time-reversal MUSIC for two-dimensional thin PEC scatterers," IEEE Geoscience and Remote Sensing Letters, vol. 11, pp. 1163-1167, June 2014.

[26] A. V. Oppenheim, R. W. Schafer, and J. R. Buck, Discrete-time signal processing. Englewood Cliffs: Prentice-hall, 1989.

[27] F. Costen, J.-P. Bérenger, and A. Brown, "Comparison of FDTD hard source with FDTD soft source and accuracy assessment in debye media," IEEE Trans. Antennas and Propagat., vol. 57, pp. 2014-2022, 2009.

[28] G. Lerosey, J. de Rosny, A. Tourin, and M. Fink, "Focusing beyond the diffraction limit with far-field time reversal," Science, vol. 315, no. 5815, pp. 1120-1122, 2007. 
Victor C. Odedo received the BEng degree in electrical and electronic engineering and the M.Sc. degree in communication engineering from The University of Manchester, Manchester, U.K., in 2010 and 2011, respectively, where he is currently pursuing the Ph.D. degree in electrical and electronic engineering. His current research interests include computational electromagnetics, microwave imaging and detection, radar-based signal processing and high-performance computing.

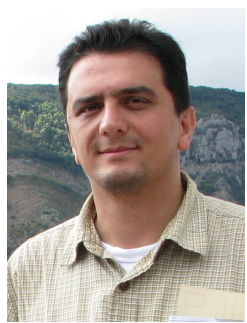

Mehmet E. Yavuz received the B.S. degree from the Middle East Technical University (METU), Ankara, Turkey in 2001, M.S. degree from the Koç University, Istanbul, Turkey in 2003 and the Ph.D. degree from the Ohio State University (OSU) in 2008, all in electrical engineering. He has worked as a Principle Scientist at the Halliburton Energy Services in Houston, TX, as a Visiting Assistant Professor at the Electrical Engineering Department of the American University of Sharjah in the U.A.E and as a Research Scientist at Intel Corporation in Hillsboro, Oregon. His current research interests include computational electromagnetics for industrial applications, radar-based signal processing and microwave imaging and detection.

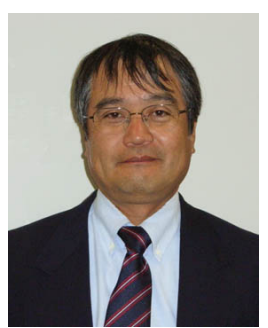

Ryutaro Himeno received his Doctor of Engineering degree from the University of Tokyo in 1988 In 1979, he joined Central Research Laboratories, Nissan Motor Co., Ltd., Yokosuka, Japan, where he has been engaged in the research of applying Computational Fluid Dynamics analysis to the car aerodynamic development. In 1998, he joined RIKEN and is the director of Advanced Center for Computing and Communication and had been the deputy program director of the Next Generation Computational Science Research Program at RIKEN till April, 2013. He is also a visiting professor at Hokkaido University, Kobe University and Tokyo Denki University. He currently studies Computational Bioengineering, High Performance Computing and blood flows of human bodies. He was a winner of Nikkei Science, Computer Visualization Contest in 2000 and Scientific Visualization Contest in 1996, and received JSME Computational Mechanics Division Award in 1997 and JSME Youth Engineer Award in 1988. He has also received the Paper Award by NICOGRAPH in 1993, Giga FLOPS Award by CRAY Research Inc. in 1990 and other awards.

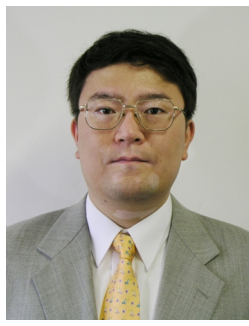

Hideo Yokota received his Doctor of Engineering degree from the University of Tokyo in 1999. In 1993, he joined Higuchi Ultimate Mechatronics Project, Kanagawa Academy of Science and Technology, Kawasaki, Japan. In 1999, he joined RIKEN and is the contract researcher of Computational biomechanics unit. 2004-2012 Bio-research Infrastructure Construction Team Leader, VCAD system research program, RIKEN. 2007-2012 Cell-scale Research and Development Team Leader,Research Program for Computational Science, RIKEN. 2013present Image Processing Research Team Leader, Center for Advanced Photonics,RIKEN. He is also a visiting Professor at Hokkaido University, Kobe University, Tokai University and the Tokyo University of Agriculture and Technology. He is currently involved in biomedical imaging, image processing and biomedical simulation. Bioimaging Society, Best Image Award, 2005. The Commendation for Science and Technology by the Minister of Education, Culture, Sports, Science and Technology, Young Scientists Prize in 2008. 